\title{
TTR
}

Traduction, terminologie, re?daction

\section{La faute de sens en traduction}

\section{Jeanne Dancette}

Volume 2, numéro 2, 2e semestre 1989

L'erreur en traduction

URI : https://id.erudit.org/iderudit/037048ar

DOI : https://doi.org/10.7202/037048ar

Aller au sommaire du numéro

Éditeur(s)

Association canadienne de traductologie

ISSN

0835-8443 (imprimé)

1708-2188 (numérique)

Découvrir la revue

Citer cet article

Dancette, J. (1989). La faute de sens en traduction. TTR, 2(2), 83-102.

https://doi.org/10.7202/037048ar

Tous droits réservés @ TTR: traduction, terminologie, rédaction — Les auteurs, d'utilisation que vous pouvez consulter en ligne.

https://apropos.erudit.org/fr/usagers/politique-dutilisation/ 


\title{
La faute de sens en traduction
}

\author{
Jeanne Dancette
}

\section{Introduction}

Si la compréhension est considérée par la plupart des théoriciens et des enseignants de la traduction comme la condition sine qua non de la traduction, son contraire, l'incompréhension est un sujet quasi tabou. Lorsqu'ils parlent d'intraduisibilité, bon nombre d'auteurs (Steiner, Mounin) font généralement référence à l'incapacité du traducteur à faire passer, dans le texte d'arrivée, ce qu'il comprend et "savoure " du texte de départ; de plus, ces auteurs appliquent généralement la notion d'intraduisibilité à des types spécifiques de textes ou à des modes particuliers d'expression (littérature et en particulier poésie, publicité, jeux de mots, quiproquos, etc.)

Pour nous, les véritables cas d'intraduisibilité naissent, très prosaïquement, de situations où le traducteur ne comprend pas ou comprend mal le message qu'il doit traduire ; or ces situations sont assez fréquentes et graves pour qu'on s'y arrête. Une analyse théorique des processus de compréhension (qui reste à faire) devrait permettre d'expliquer les niveaux et les degrés de la compréhension.

Dans cette optique, cet article sera consacré à une discussion de la faute de sens et des notions de faux sens, contresens et non-sens, encore trop souvent utilisées, à la manière des sévères pédagogues des versions grecques et latines, comme des condamnations rédhibitoires, laissant l'apprenant ou l'apprenti-traducteur dans une certaine confusion.

Dans les ouvrages pédagogiques sur la traduction, les phénomènes de la déviation de sens ont été, pourtant, maintes fois observés; nous 
ne mentionnerons, pour la période contemporaine, que Vinay et Darbelnet (1968), Maillot (1969), Gouadec (1974), Bénard et Horguelin (1979), Delisle (1984), parmi tant d'autres. Mais leurs observations, pour intéressantes qu'elles soient, se placent souvent «en aval» de la faute de sens, c'est-à-dire la faute de sens une fois commise; elles ne permettent donc pas de remonter suffisamment à la source, qui est soit l'incompréhension du texte de départ, ou de ses segments, soit l'insuffisante maîtrise de la langue d'arrivée. Or, ces deux causes majeures de la «faute» de traduction sont à distinguer, car, même s'il est souvent vrai que "forme et sens sont en relation dialectique et, à ce titre, sont indissociables " (Larose $1989: 209$ ), nous pensons indispensable d'en faire l'analyse en tant que phénomènes distincts.

Il a fallu attendre la vague des études empiriques sur la traduction, entreprises le plus souvent dans un cadre psycho-linguistique et souvent dans l'optique de l'apprentissage de la langue seconde (Lörscher 1987, Krings 1986, Dechert et Sandrock 1986 en Allemagne, Séguinot 1989 au Canada, Tirkkonen-Condit 1987 en Finlande, entre autres) pour que l'on s'intéresse sérieusement à la faute de sens dans sa genèse, à sa source et à sa nature.

Nous proposons de centrer l'analyse de la compréhension de texte sur l'articulation entre les différents composants linguistiques -morphologique, syntaxique, sémantique -, d'une part, et, d'autre part, sur l'articulation entre la saisie du sens littéral et les interprétations à en faire en raison du contexte verbal et du contexte d'énonciation. Nous rappelons la distinction entre sens (ce à quoi renvoie un mot dans un système abstrait de la langue) que nous appelons aussi, dans cet article, sens littéral, et signification (ce à quoi renvoie le signe lorsqu'il s'insère dans l'énoncé issu d'un acte de parole), que nous appelons aussi sens contextuel.

Nous verrons, dans une première partie, les grandes lignes d'un cadre théorique permettant de situer l'analyse des fautes de compréhension et, dans une deuxième partie, l'analyse de quelques fautes de compréhension commises sur un texte à traduire.

\section{Méthode de recherche}

Cet article fait état d'analyses élaborées à partir d'un texte dont nous reproduisons ici deux paragraphes. (Le texte entier figure à l'annexe.) Il s'agit d'un extrait d'article de journal paru dans World Press Review en décembre 1988, après les élections américaines. Un groupe de 20 répondants, étudiants de maîtrise en traduction de l'Université de Montréal, devait répondre à des questions de compréhension et traduire ces deux paragraphes:

It is easy to be wise after an event. But the Jackson people can say with justification that if their man had been white, he might have pulled 
it off with what top adviser Bob Borosage of the Institute for Policy Studies calls his « updated version of the populist strain in the Democratic Party politics» - bringing out non-voters and the excluded with an assault on the "economic royalists. " Jackson's reception among white, blue-collar workers shows what can be done, says Borosage.

In the post-mortem that has already started, the Democrats' right wing will press for further moderation and accommodation of the social and foreign policy prejudices of Southern white males and « Reagan Democrats." A candidate for 1992 who fits that bill might be Sen. Sam Nunn or Sen. Albert Gore, who did well on Super Tuesday but proved to be no Crocodile Dundee in the jungles of New York.

Ce texte nous fournira les exemples illustrant les points théoriques de notre première partie. Nous l'utiliserons aussi dans la deuxième partie pour exposer et analyser les problèmes de compréhension qu'il a posés à nos répondants.

\section{Les grandes lignes du cadre théorique}

Les théories que nous avons retenues relèvent toutes d'une approche logique privilégiant, dans l'acquisition des connaissances et dans la compréhension des messages, les opérations analytiques. Ainsi, la compréhension du texte s'élaborerait, entre autres processus, par le résultat conjugué des analyses linguistiques et des activités cognitives d'inférence et de déduction opérant sur des structures.

Nous ne pensons pas, cependant, que cette position rende compte de tous les mécanismes de compréhension. La position selon laquelle la compréhension se produirait aussi de façon synthétique, non analytique, nous semble tout à fait défendable. Ainsi, les évocations, déclenchées par les mots de façon associative, de même que les interprétations globales de phrases, ne nous semblent pas être des phénomènes négligeables dans l'acquisition des connaissances et de la compréhension.

Nous faisons l'hypothèse, néanmoins, que l'approche logique est communément utilisée par les locuteurs (et interlocuteurs) d'une langue pour traiter les signes linguistiques. Par ailleurs, elle se prête relativement bien à une modélisation. Les formalisations auxquelles cette approche donne lieu (structures, arbres, réseaux) permettent de représenter ce qui pourrait être un «calcul » sur le sens et surtout, en ce qui concerne particulièrement notre sujet, elles rendent facile la comparaison des représentations sémantiques et conceptuelles. On pourra ainsi établir plus précisément la fidélité au sens par confrontation de réseaux, de structures et de graphes. Mais bien entendu, notre modèle, comme tout modèle, n'est qu'une simplification de la réalité. ${ }^{1}$

1. Les théories psychologiques et neurolinguistiques (approche connexionniste) posent d'autres postulats qu'il serait difficile d'intégrer dans cet article, vu les contraintes d'espace. Nous n'en tiendrons donc pas compte. 
Les formalisations permettent de mettre en évidence la structure d'une séquence de mots et de phrases. En effet, les «calculs» de sens que l'on fait quand on analyse un message pour le traduire ou qu'on se livre à des opérations proches de la traduction (paraphrasage, adaptation, contraction de texte, comparaison et évaluation de traductions) s'effectuent, pensons-nous, au moins en partie, sur la base de représentations syntaxico-sémantiques et de représentations des connaissances, organisées en structures (non pas superposées mais concentriques). ${ }^{2}$

Nous analyserons d'abord le sens littéral - réalisé au niveau linguistique - et ensuite le sens contextuel, tel qu'il est précisé ou modifié au cours des interprétations rendues nécessaires par le contexte verbal et la situation ${ }^{3}$. Du fait que les processus de compréhension humaine ne suivent pas une séquence établie d'avance qui irait, par exemple, comme dans les modèles de traduction automatique et d'intelligence artificielle, du linguistique (en passant par les différentes étapes : morphologique, syntaxique, sémantique et textuelle) au cognitif, mais s'interpénètrent à tous les niveaux, nous chercherons non pas à décrire les opérations faites à chaque niveau par le lecteur, mais à dégager les interactions entre les différents niveaux telles qu'elles pourraient se produire à la lecture de phrases constituant un message.

\subsection{Le sens littéral}

Pour passer d'une phrase à sa représentation, il faut effectuer plusieurs analyses de type linguistique :

L'analyse syntaxique permet d'étudier chaque mot, par l'examen de ses traits morphologiques, de sa catégorie et de sa fonction grammaticales; le résultat peut être représenté sous forme d'arbre syntaxique, par une règle de réécriture $\mathrm{P}-\mathrm{SN}+\mathrm{SV}$ (où $\mathrm{P}$ est le symbole de la phrase; SN, le symbole du syntagme nominal et SV, le symbole du syntagme verbal).

2. Le caractère distinct de ces différentes structures est loin d'être démontré ; pour certains auteurs (Jackendoff, 1985), la représentation sémantique en particulier n'est pas autonome : "semantic and conceptual structure collapse into a unified level, and syntactic form is mapped by the correspondence rules directly into conceptual structure, without an intermediate level that accounts for purely linguistic inference." (p. 105)

3. Nous utilisons le terme sens contextuel plutôt que ceux de sens cognitif, ou sens psychologique (De Beaugrande, 1980), qui introduisent quelque confusion. Nous ne retenons pas l'opposition sens objectif/sens subjectif car nous ne pensons pas que le sens dit «subjectif" le soit complètement, mais qu'il relève de règles d'élaboration plus difficiles à dégager à cause de leur caractère particulier ; ni que le sens dit "objectif " le soit complètement, même s'il relève de règles d'élaboration plus générales. 
À cette étape, les ambiguïtés syntaxiques peuvent être vues, comme le montre l'exemple tiré de notre texte: who did well on Super Tuesday... L'ambiguïté possible vient du manque de précision morphologique du prétérit anglais (singulier ou pluriel); elle se traduirait par deux arbres syntaxiques différents.

L'analyse lexico-sémantique permet de préciser le sens de la phrase, composé des significations possibles (dans le contexte) des lexèmes et des rapports syntaxiques dégagés par l'analyse syntaxique. Mais, étant donné qu'un même lexème peut avoir plusieurs significations différentes selon le contexte, cette analyse ne peut réussir que lorsque la plausibilité d'une signification possible est vérifiée par le contexte. Si elle est rejetée, une autre hypothèse sur la signification devra être envisagée jusqu'à ce qu'il y ait concordance entre les différents niveaux d'analyse linguistique et extralinguistique. Ainsi, la phrase a) suivante

a) The right wing will press for further moderation and accommodation of the social and foreign policy prejudices of Southern white males and Reagan Democrats.

pourrait être analysée syntaxiquement de façon à placer sous la même tête syntagmatique moderation et accommodation, d'où dépendrait le syntagme prépositionnel of the social and foreign policy prejudices of... Mais l'analyse lexico-sémantique permet de remettre en question ce premier découpage syntaxique, car on peut voir qu'il y aurait une incohérence à demander à la foi «moderation of prejudices » et « accommodation of prejudices ». En envisageant les sens possibles de moderation et d'accommodation, et en cherchant quels sont les arguments de ces prédicats, on peut arriver à rectifier la représentation syntaxique faite initialement.

Cette phrase s'est avérée très difficile à comprendre pour nos répondants. Il semble que l'affinité sémantique des mots ne suffisait pas pour la désambiguïser; elle nécessitait, pour être comprise, en plus de connaissances linguistiques précises, de l'information de sens commun, non directement liée au sens des lexèmes, mais plutôt à notre connaissance du monde. (Voir plus loin la notion de «frame».)

\subsubsection{L'articulation entre la syntaxe et la sémantique}

Nous nous appuyons, pour cette section, sur les théories de la sémantique générative, ainsi que sur les théories de l'intelligence artificielle et de la traduction automatique, issues pour la plupart de la grammaire générative. Leur point commun est la recherche de la correspondance entre la structure de surface et le sens. Il s'agit de trouver les règles sémantiques qui s'associent à chaque représentation syntaxique et qui en conditionnent l'application. 
Nous trouvons particulièrement utiles les théories qui visent à expliciter les rôles sémantiques des différents éléments de la phrase. Les cas de Fillmore (1968) permettent de mettre en évidence, à la manière des cas syntaxiques, les relations de sens entre les noms et les verbes (ou verbes nominalisés) à l'intérieur d'une proposition. Le verbe est alors vu comme un prédicat logique auquel sont associés des arguments.

Ainsi, en reprenant la phrase a), le premier découpage syntaxique mène à l'interprétation, fréquente chez les répondants, selon laquelle moderation, placé sur le même plan que accommodation, aurait pour argument sujet Southern white males and "Reagan Democrats", et pour argument objet social and foreign policy prejudices. La représentation sémantique correcte n'attribue aucun de ces arguments-là au prédicat moderation, mais tient compte de l'ellipse des arguments ; des connaissances pragmatiques permettent de supposer comme argument sujet possible "candidat futur" et comme argument objet possible "programme politique".

Les modèles qui font la relation entre la grammaire des cas et les réseaux sémantiques sont encore plus intéressants pour la traduction, car ils s'appliquent à des phrases entières, voire à des textes (Mel'čuk 1978, 1981, de Beaugrande, 1980).

Du point de vue formel, une structure sémantique est un réseau ou un graphe dont les points sont reliés; les sommets ou les nœuds ont pour étiquette les unités sémantiques, c'est-à-dire les sens. Mel'čuk distingue deux grandes classes d'unités sémantiques: 1) les FONCTEURS qui se subdivisent en prédicats (relations, propriétés, actions, états, événements, etc.), en connecteurs logiques ( «si», «et», «ou», "ne...pas ") et en quantificateurs ( «tout", "il existe», etc.) ; et 2) les NOMS (DE CLASSES) D'OBJETS, comprenant les noms propres. Seuls les foncteurs peuvent être à l'origine des flèches ou des arcs, qui sont orientés du foncteur à ses arguments. (Pour être complète, la représentation sémantique devrait se doubler de la structure sémantico-communicative qui spécifie les intentions du locuteur en s'attachant à l'organisation du message. Elle montre, par exemple, les oppositions suivantes : thème/rhème, vieux/nouveau, mis au premier plan/mis à l'arrière-plan.)

Une représentation sémantique, formalisée par un réseau sémantique, peut produire un nombre incalculable de paraphrases qui auraient toutes le «même» sens.

\subsubsection{Articulation entre les niveaux lexical, syntaxique et sémantique}

Le modèle de Mel'čuk prévoit la production d'une représentation du sens des lexèmes sous forme de réseaux sémantiques. De tels réseaux 
s'intègrent dans le réseau sémantique de la phrase, et rendent compte de l'articulation entre le niveau lexical et le niveau syntaxicosémantique.

Ce modèle nous paraît adéquat pour décrire les opérations effectuées par le traducteur, souvent appelé à préciser le sens d'un lexème. Or, comme le sens d'un lexème est rarement entièrement couvert par la signification donnée par les dictionnaires courants, et que son décodage dépend souvent de la structure sémantique de la phrase (et du message), la découverte du sens résultera d'une analyse s'effectuant au moins à ces deux niveaux - lexical et sémantique -, en postulant le sens. Ainsi, la description sémantique du lexème, doté de ses caractéristiques syntaxiques et sémantiques, peut donner la clé à la mise en évidence de la structure sémantique de la phrase et réciproquement la structure sémantique peut aider à préciser le sens d'un lexème.

\subsection{Articulation entre le niveau linguistique et le niveau cognitif}

Nous sommes partie de l'hypothèse que le sens d'un texte se dégage de la conjonction du sens littéral de ses composants et des interprétations que le contexte discursif conduit à faire.

Les théories qui cherchent à dégager les règles d'élaboration de la signification en contexte de communication sont nombreuses et diverses, mais elles se heurtent toutes à la complexité de la tâche et n'ont pas encore réussi à présenter une formalisation intégrant toutes les dimensions de la compréhension de texte.

Cependant, nous trouvons extrêmement utiles, pour l'analyse des processus de compréhension en traduction, deux types d'études, celles centrées sur la théorie de la macrostructure (Kintsch et van Dijk 1975, 1983, entre autres), et celles des théories des schémas ( "frames" de Minsky, 1974) et autres structures qui en ont découlé, scénarios, scripts, plans, etc.

\subsubsection{Théories de la macrostructure}

Leur intérêt a été bien étudié par Larose (1989) qui les intègre dans son modèle de la traduction et en fait une application pour l'évaluation des traductions. Nous les retenons aussi pour leur valeur explicative de la compréhension.

Kintsch et van Dijk ont montré, par des expériences de rappel d'histoires et de résumés, le rôle des propositions ${ }^{4}$ dans la compréhension. Ils s'appuient sur l'hypothèse que la compréhension s'élabore et

4. À ne pas confondre avec la proposition grammaticale («clause " en anglais). 
se manifeste, entre autres choses, dans la formation d'une suite cohérente de propositions, appelée base de texte. Le lecteur, disent-ils, traite le texte qu'il reçoit en en arrangeant le contenu sous forme de propositions hiérarchisées.

Les auteurs définissent ainsi la proposition:

... a proposition is usually taken as the meaning of a (declarative) sentence. This meaning has a composite nature : It is a construction of the meanings of the component expressions - the words or phrases of the sentence. This construction is the output of the semantic interpretation rules, and follows the well-known Fregean principle of functionality: The interpretation of composite expressions is a function of the interpretation of its component expressions. (1983: 112)

Les propositions ne sont pas nécessairement données telles quelles par le texte, mais peuvent être construites par le récepteur du texte à l'aide de trois catégories de règles correspondant à des opérations de généralisation (substitution, à partir de l'information présente, d'une proposition donnée par une autre plus générale), de suppression (d'éléments d'information redondants ou considérés comme non pertinents) et d'intégration ou construction (d'information d'ordre supérieur).

La représentation sémantique du discours est alors définie comme un n-tuple de propositions. "De façon intuitive, la signification d'une proposition ou d'une phrase se définit par la séquence des propositions (et de leurs liaisons) qu'elle exprime." (1975: 99).

En appliquant cette définition, la signification de la deuxième phrase de notre texte serait donnée par la liste des propositions extraites (par inférence et déductions) et des relations qui les lient les unes aux autres :

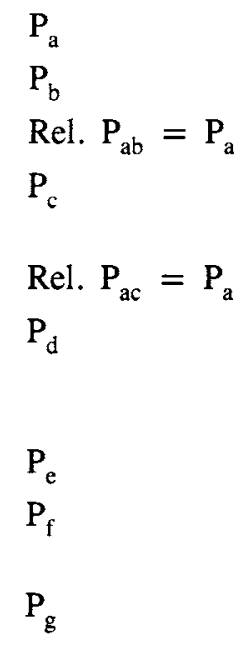

Jackson aurait pu gagner

Jackson est noir / pas blanc

malgré $\mathrm{P}_{\mathrm{b}}$

Jackson a quelque chose (de positif) (programme ou style politique)

grâce à $\mathrm{P}_{\mathrm{c}}\left(\mathrm{P}_{\mathrm{c}}\right.$ instrument de $\left.\mathrm{P}_{\mathrm{a}}\right)$

Bob Borosage qualifie le quelque chose en $P_{c}$ de « version moderne du populisme du Parti démocrate»

Bob Borosage est conseiller principal

Bob Borosage travaille à l' 'Institute for Policy Studies"

Jackson aurait fait voter les (habituels) abstentionnistes et exclus de la société 


$$
\begin{array}{ll}
\text { Rel. } \mathrm{Pag}=\mathrm{P}_{\mathrm{a}} & \begin{array}{l}
\text { du fait de } \mathrm{P}_{\mathrm{g}} \\
\mathrm{P}_{\mathrm{h}}
\end{array} \\
\begin{array}{l}
\text { Il y a /aurait une attaque, lancée par } \\
\text { Jackson, contre les «royalistes de l'éco- } \\
\text { nomie» }
\end{array} \\
\text { Rel. } \mathrm{P}_{\mathrm{gh}}=\mathrm{P}_{\mathrm{g}} & \begin{array}{l}
\text { à cause de } \mathrm{P}_{\mathrm{h}} \text { (où } \mathrm{P}_{\mathrm{h}} \text { rend possible } \mathrm{P}_{\mathrm{g}} \text { ) } \\
\mathrm{P}_{1}
\end{array} \\
\begin{array}{l}
\text { Les partisans de Jackson ont raison de } \\
\text { dire }\left(\mathrm{P}_{\mathrm{ab}}\right)
\end{array}
\end{array}
$$

L'utilité pratique de cette approche est évidente pour notre propos. Toute traduction qui ne restituerait pas intégralement et fidèlement chaque élément d'information contenu dans la liste des propositions serait insatisfaisante. Malgré la part de subjectivité dans la forme et le nombre des propositions (que reconnaissent les auteurs, 1983), la détermination de l'erreur de sens serait aisée à faire et convaincante. De plus, la portée de l'erreur serait facile à établir car les propositions peuvent être hiérarchisées par réduction du texte à sa macrostructure ${ }^{5}$, ou pour reprendre les termes de Larose, «la gravité d'une faute (...) sera donc d'autant plus grande qu'elle occupera un niveau élevé de pertinence dans le texte" (1989: 237). (Nous émettons une seule réserve face à ce type de position: la gravité de l'erreur peut être indépendante de sa portée macrostructurelle (place dans la hiérarchie) selon la fonction de la traduction. Si l'objet de la traduction est de faire faire un geste au récepteur, une erreur sur un élément très secondaire du point de vue de la macrostructure, comme pourrait l'être, par exemple, «bouton de gauche» au lieu de «bouton de droite», peut être extrêmement grave. Il ne faudrait donc pas fonder un classement des erreurs uniquement sur leur portée macrostrucurelle.)

La macrostructure, définie par les auteurs comme la «structure de signification globale d'un texte " $(1975: 101)$ résulte de l'application de règles qui permettent la projection de la base de texte sur une structure supérieure. Ces règles «doivent satisfaire au principe d'implication " (1975: 102). Nous avons hiérarchisé, à titre d'exemple, les propositions constituant la macrostructure de notre segment de texte ; pour des raisons de présentation, nous avons utilisé un style télégraphique et des abréviations.

5. La macrostructure s'inscrit généralement dans une structure de plus haut niveau, déterminée par le genre d'écrit (narratif, argumentatif, etc.), appelée superstructure. 


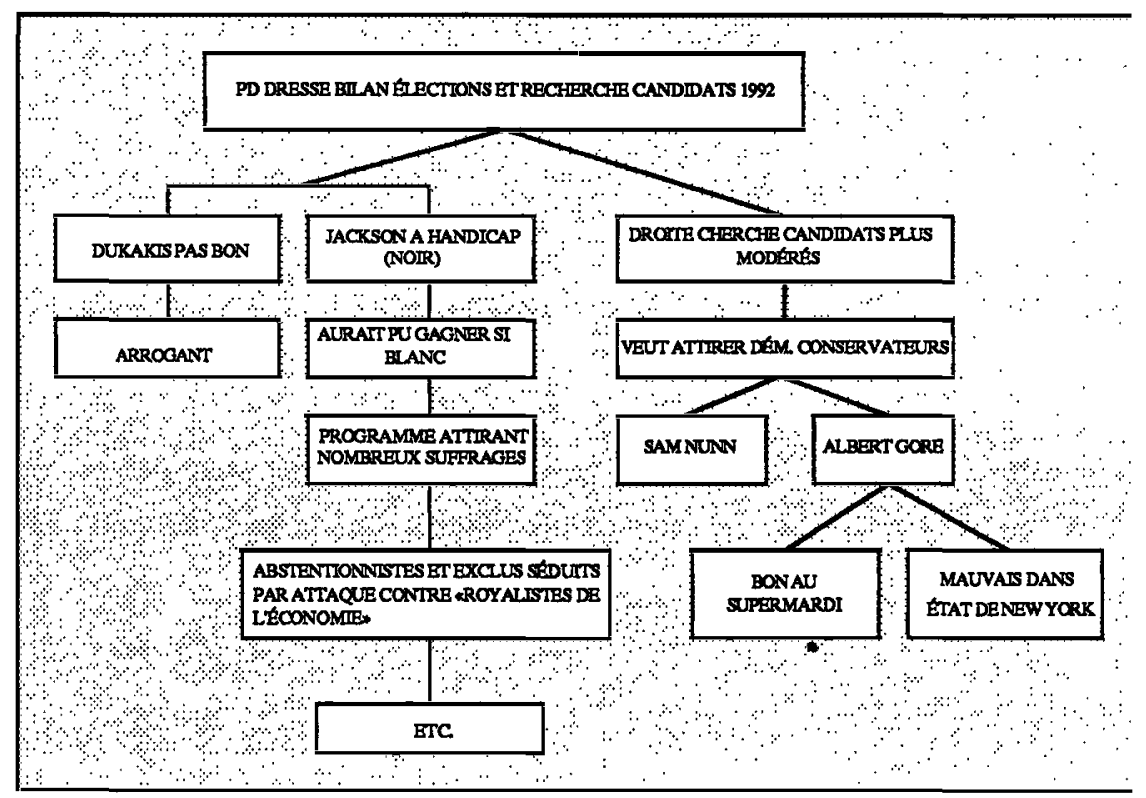

\subsubsection{Théories des représentations des connaissances (schémas, scripts, plans, etc.)}

Ces théories (Minsky 1974, Schank et Abelson 1977) postulent l'existence d'une variété de structures de mémoire complexes dans lesquelles sont emmagasinées les connaissances du monde. Nous ne rentrerons pas dans le détail, mais mentionnerons que ces notions nous paraissent utiles pour expliquer la mise en rapport des propositions données par le texte, ou inférées et déduites, avec les connaissances extralinguistiques emmagasinées, selon ces théories, dans la mémoire sous forme de schémas, plans, scripts, scénarios, etc. Le lecteur s'assure que sa représentation du texte fournit une certaine cohérence avec ses connaissances antérieures. Ces connaissances rendent possible ou facilitent sa compréhension.

Il faut bien voir, comme Kintsch et van Dijk le montrent, l'interpénétration du pragmatique et du sémantique. Ainsi, dans notre exemple, l'analyse lexico-sémantique de moderation and accommodation ne permettait pas à coup sûr de voir et éventuellement de résoudre l'ambiguïté syntaxique ; cependant, le recours aux connaissances extralinguistiques et pragmatiques permettait de choisir, parmi les sens possibles et les combinaisons syntagmatiques possibles, ceux qui étaient compatibles avec la réalité du monde de référence. 
Le schéma d'organisation politique (dans le régime social dans lequel s'insère le thème du texte) comprendrait, entre autres, les informations suivantes :

Un parti a une politique (un programme) qui le distingue des autres

Un parti a une clientèle

Un parti cherche à faire élire ses candidats à des postes

On adhère à un parti

Il peut exister, à l'intérieur d'un parti, différentes tendances qui prennent position sur des aspects particuliers de la politique, dont la politique économique et sociale, les affaires extérieures, etc.

Les différentes tendances s'affrontent à l'intérieur du parti et veulent dominer

La droite tire vers une politique plus conservatrice; la gauche, vers une politique plus radicale

etc.

Ainsi, la compréhension d'un texte suppose, selon ces théories, la mise en œuvre de plusieurs mécanismes successifs, dont la reconnaissance des schémas, scénarios, etc. invoqués par le texte et le remplissage des informations manquantes mais contenues dans la structure de connaissances. La compréhension consiste alors à saisir les relations temporelles (ex. : pour être élu il faut avoir reçu l'appui de divers groupes), à appliquer des connaissances antérieures que l'on a sur des personnages (Jackson, Dukakis) et sur des objets (élections, campagne), à inférer correctement des faits non exprimés; cette faculté permet, par exemple, de trouver l'antécédent des pronoms he et de l'adjectif possessif his dans la deuxième phrase de notre texte; et de formuler des attentes sur des éléments d'information à venir selon ce qui a déjà été compris (ex. : la droite, critique de Jackson, cherchera des candidats plus modérés).

En conclusion à cette première partie, on peut dire que la faute de compréhension peut être due à une série de facteurs :

1) mauvais décodage linguistique : mauvaise analyse morphologique, mauvaise analyse syntaxique (mauvais découpage), méconnaissance du lexique, mauvaise analyse sémantique ;

2) erreurs dans les opérations cognitives : construction de mauvaises inférences, absence de connaissances préalables permettant de reconstruire le non-dit (ellipses, sens vague, flou dans l'expression, métaphores), construction de présupposés erronés.

Mais il est important de bien voir l'interaction entre les deux domaines de connaissances qui sont liés par un étroit rapport de 
complémentarité et de compensation. Avec de faux présupposés on peut faire faire des entorses à la syntaxe, au lexique, à la sémantique ; ou, au contraire, malgré de faux présupposés, le décodage linguistique peut permettre de construire un sens exact et, en cours de route, de modifier la première hypothèse.

Dans la deuxième partie, nous proposons l'analyse de quelques fautes de compréhension commises à propos du texte. Cette analyse nous permettra, peut-être, d'éclairer davantage la notion de fidélité du message.

\section{Analyse de quelques erreurs de compréhension}

\subsection{Liste des éléments textuels qui ont causé une faute de compréhension}

Nous avons classé par niveau d'analyse tous les éléments textuels sur lesquels des erreurs ont été commises par nos répondants. ${ }^{6}$

Pour ne pas augmenter indûment la longueur de cet article, nous ne donnerons pas la liste complète de tous les éléments, mais seulement des exemples pour chaque catégorie de notre typologie. Nous nous attacherons davantage aux remarques qu'elle nous conduit à faire. Mentionnons que nous ne cherchons pas ici à identifier la cause de l'erreur mais son lieu précis dans le texte.

a) Code typographique ex. : l'abréviation Sen. (Ne reconnaissant pas l'abréviation, des répondants ont interprété Sen. comme désignant un prénom ou surnom, suivi d'un point de fin de phrase, ce qui entraînait des problèmes syntaxiques rejaillissant sur toute la phrase.)

b) Morphologie ex, : policy (substantif au lieu d'adjectif), who (did well...Dundee)

c) Lexique - mots et expressions idiomatiques ex. : wise, strain, accomodation, to pull it off, to fit the bill

d) Utilisation du contexte pour le choix du sens des mots et expressions ex. : to bring out, reception, assault

e) Utilisation du contexte pour la définition des relations syntaxico-sémantiques ex. : with (an assault) : instrument de bringing out ; assault: prédicat dont l'argument sujet est «Jackson»; moderation

6. Cette liste est représentative du niveau des répondants; elle serait plus longue s'ils avaient une moins bonne connaissance de l'anglais ou du thème du texte à traduire. 
f) Utilisation des connaissances extralinguistiques (notionnelles ou pragmatiques) ex. : Democratic Party, populist, "economic royalists », Super Tuesday

Sur les six catégories de ce classement, trois relèvent des connaissances linguistiques; et trois, de la performance du récepteur. Sur l'ensemble des vingt-trois traductions que nous avons analysées, nous avons relevé un total de 31 éléments textuels qui ont été le lieu d'une erreur de compréhension. Sur ces 31 éléments, 14 relèvent des connaissances linguistiques et 17 , de la performance du récepteur. Nous pensons qu'il est important, pour les professeurs de traduction, de s'arrêter sur ce rapport. S'il est vrai qu'il dépend beaucoup de la nature du texte, en plus des compétences des étudiants, il indique toutefois quelque chose sur la nature de certains problèmes de la traduction.

Cette distinction entre connaissances linguistiques et performance du récepteur - que le classement des fautes trouvées dans notre corpus nous a conduite à établir - justifie pleinement, à notre sens, la distinction entre sens littéral et sens cognitif (ou signification $=$ sens + interprétations), et en montre la complémentarité.

Ainsi, des éléments textuels peuvent se retrouver à plus d'un niveau d'analyse, comme le montrent les exemples suivants :

- bringing out figure en d) pour le sens contextuel de l'expresssion et en e) pour la relation interpropositionnelle avec pulled it off;

- assault figure en d) pour le sens du mot et en e) pour les rôles sémantiques qui déterminent l'argument sujet de assault.

- accommodation figure en c) et en e) pour la détermination des arguments du prédicat.

La superposition des difficultés que ce classement met en évidence permet d'observer les mécanismes de compensation, ou « complémentarité " et de "supplétion " que nous définirons comme la faculté, en cas d'incertitude, à recourir à un autre niveau pour tester des hypothèses (complémentarité) et, en cas de problème, de recourir à un autre niveau pour trouver la solution (supplétion). Une lacune au niveau linguistique, lexical par exemple, pourra être compensée par la faculté à appliquer des connaissances extralinguistiques ; inversement, cette même lacune peut être aggravée par une moins grande aptitude à utiliser les connaissances extralinguistiques.

Par exemple, dans le syntagme populist strain, le sens de populist peut être inconnu du traducteur, mais l'inférence (facteur facilitateur) que c'est un terme du langage politique lui fera vraisemblablement décider (à juste raison) de garder le mot-sosie " populiste "; par contre, si le sens de strain est incorrectement décodé comme signifiant "pression, contrainte», et qu'à cette lacune de vocabulaire s'ajoute une 
mauvaise connaissance du thème (facteur inhibiteur), alors on peut facilement expliquer l'erreur de sens «pression exercée par le peuple». (Cette faute mène à un non-sens car la phrase ne présente plus de cohérence par rapport au monde de référence du texte.)

Vice versa, une lacune au niveau de la performance textuelle ou au niveau des connaissances extralinguistiques peut être suppléée par une bonne compétence linguistique. Ainsi, sans connaître ni Sam Nunn ni Albert Gore, ni ce qu'est le "Super Tuesday", ni le personnage de "Crocodile Dundee", des répondants ont été capables de traduire, de manière certes prudente mais correcte, la proposition who did well on Super Tuesday but proved to be no Crocodile Dundee in the jungles of New York.

Nous n'utilisons pas la notion traditionnelle d'unité de traduction car elle nous paraît beaucoup trop floue et imprécise dans une tentative d'explication de la faute de sens. Si l'on prend, en effet, les unités de traduction comme des «éléments micro-textuels " (Larose, 1989: 208), le nombre de ces éléments peut être si élevé que la notion perd de sa valeur pratique. Si on les prend comme des unités de traitement (cf. de Beaugrande, 1980), alors la notion devient très subjective. Ainsi, l'unité moderation devrait être décomposée en plus petites parties pour rendre compte de la complexité des analyses rentrant en ligne de compte de manière plus ou moins consciente dans les processus de compréhension. Inversement, ne pourrait-on pas dire qu'une suite linguistique telle que bringing out the non-voters and the excluded with an assault on constitue, au niveau des relations de sens entre les mots de la phrase, une seule et même unité de traduction? Le traducteur ne peut pas la traiter autrement que globalement au niveau des relations de sens, même s'il la décompose au niveau du choix du vocabulaire dans la langue d'arrivée. L'unité de traduction serait donc une unité à extension variable. Comment utiliser la notion, par exemple, pour décrire un contresens du genre « inciter ... à voter contre les royalistes économistes "? Quelle est l'unité de traduction fautive, with ou assault ? Ces considérations nous ont conduite à préférer au concept d'unité de traduction, dont la définition est souvent subjective dans son application (puisqu'elle dépend de la façon dont le traducteur traite les mots et groupes de mots), celui, visible, d'éléments textuels, tels que nous les avons exposés plus haut.

\subsection{Analyse de traductions fautives}

Nous faisons l'hypothèse que, parmi les fautes de sens, les plus graves, pour un texte de ce genre, informatif et argumentatif, sont celles qui mettent en cause les relations syntaxiques et sémantiques. Leur portée peut s'étendre sur une ou plusieurs propositions et la faute peut se répercuter, par effet de cascade, sur tout un passage. 
L'analyse d'un extrait de traduction nous permettra de voir en quoi l'utilisation des représentations formelles du sens (aussi simplifiés soient-elles) permet de jeter un éclairage nouveau sur l'évaluation des traductions. Le jour où les ordinateurs seront vraiment « intelligents » et effectueront avec un minimum de risques d'erreur la traduction automatique, alors, du même coup, l'évaluation automatique des traductions deviendra facile! Même si notre texte est un cas extrême mettant à rude épreuve l'intelligence des modèles cybernétiques, ce jour n'est peut-être pas si loin.

Traduction fautive :

Mais les supporters de Jackson peuvent prétendre sans se tromper que si leur candidat avait été blanc, il aurait pu ce que Bob Borosage de l' 'Institute for Policy Studies" appelle une "version moderne du courant populiste les politiques du parti démocratique - les non-votants et les exclus et la forteresse économique des royalistes".

Ce passage contient principalement deux fautes de compréhension.

La première est un exemple typique de ce qu'on appelle traditionnellement un contresens. Le sens de l'expression idiomatique pulled it off est inconnu (niveau c) ; et cela entraîne une cascade de fautes:

- une faute de relations (niveau e). With devient argument du prédicat pulled it off, au lieu d'être un syntagme prépositionnel ;

- une faute d'interprétation contextuelle (niveau $\mathrm{f}$ ). "Version du populisme $»$ devient quelque chose de négatif et entraîne le choix du mot sévissant; c'est un deuxième contresens par ricochet.

La portée du contresens peut être visualisée au moyen des graphes :
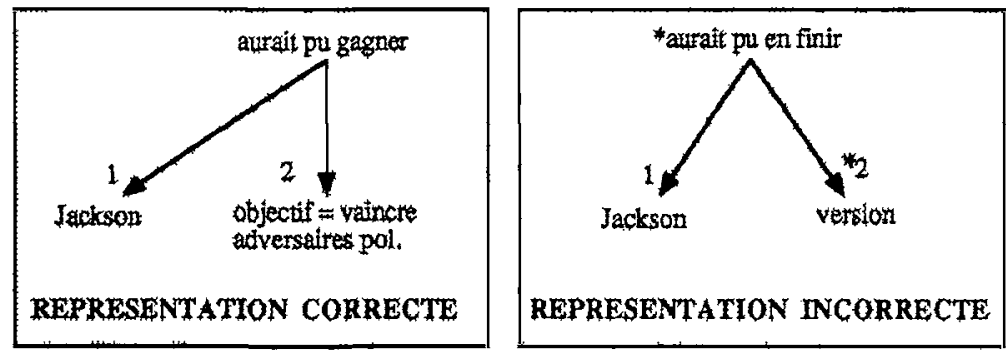

Comme c'est souvent le cas, le contresens est, ici, le double résultat d'une erreur lexicale (valeur sémantique du nœud correspondant à pulled it off) et d'une mauvaise construction de relation (la flèche) 
entre deux nœuds représentant deux concepts. Ainsi, la portée de la faute est toute la proposition exprimée par le graphe.

La deuxième faute majeure est un exemple typique de faux sens, faute d'ordinaire moins grave, dit-on, que le contresens; mais il entraîne, ici, une erreur dans l'établissement des relations sémantiques qui conduit à un contresens :

- Bringing out est traduit par « réveiller»

- with (an assault) est compris comme étant une relation de coordination causative ( «Jackson faisant aller à l'assaut les abstentionnistes et les exclus»). Le graphe du prédicat «assaut» montre l'erreur :
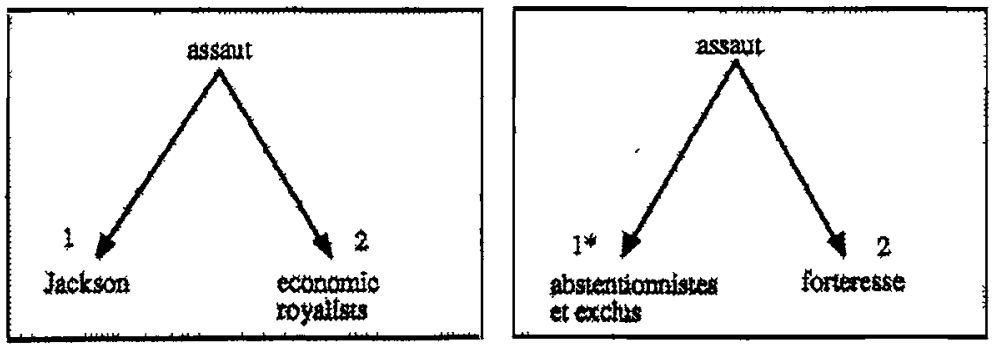

Si la nature de la faute peut être facilement visualisée au moyen des graphes, l'évaluation de la traduction peut aussi se faire par l'analyse des propositions de la traduction étudiée, que l'on peut confronter avec la liste des propositions attendues (voir plus haut en 1.2.1).

\subsection{Vérification de la portée de la faute de sens par la confrontation des propositions}

Nous reproduisons ci-dessous la liste des propositions extraites de la traduction fautive; nous avons marqué d'un astérisque les propositions qui different des propositions attendues:

$\begin{array}{ll}* \mathrm{P}_{\mathrm{a}} & \begin{array}{l}\text { Jackson aurait pu en finir avec quelque- } \\ \text { chose (de néfaste) } \\ \mathrm{P}_{\mathrm{b}}\end{array} \\ \text { Rel. } \mathrm{P}_{\mathrm{ab}} & \text { correcte } \\ * \mathrm{P}_{\mathrm{c}} & \text { devient incorrecte } \\ * \text { Rel. } \mathrm{P}_{\mathrm{ac}} & \text { absente } \\ \mathrm{P}_{\mathrm{d}} & \text { correcte } \\ \mathrm{P}_{\mathrm{e}} & \text { correcte }\end{array}$




$\begin{array}{ll}\mathrm{P}_{\mathrm{f}} & \begin{array}{l}\text { correcte } \\ * \mathrm{P}_{\mathrm{g}}\end{array} \\ * \text { Rel. } \mathrm{P}_{\mathrm{a}}{ }^{*} & \begin{array}{l}\text { Jackson aurait pu réveiller les non-votants } \\ \text { et les exclus }\end{array} \\ * \mathrm{P}_{\mathrm{h}} & \begin{array}{l}\text { relation non marquée (coordonnée) } \\ \text { Les non-votants et les exclus auraient } \\ \text { pualler à l'assaut de la forteresse }\end{array} \\ * \operatorname{Rel} . \mathrm{P}_{\mathrm{g}}{ }^{\mathrm{h}} & \begin{array}{l}\text { relation non marquée (coordonnée au lieu } \\ \text { de facilitatrice) }\end{array} \\ \mathrm{P}_{\mathrm{i}} & \text { correcte }\end{array}$

Ainsi, sur les neuf propositions qu'on aurait dû retrouver, quatre sont fausses; et sur les quatre relations qu'on attendait, les quatre sont soit fausses soit non marquées. Or, ces propositions forment le nœud de la phrase (une partie essentielle de l'argumentation). La portée de la faute est définie par la place de l'élément fautif dans la proposition et par la place de la proposition dans la macrostructure.

La fidélité de la traduction est ainsi définie par l'adéquation entre les propositions du texte original et du texte traduit, quant à leur contenu informatif.

Cette définition rejoint celle que donne Mel'čuk de l'égalité de sens. Sont synonymes toutes les phrases qui ont la même représentation sémantique.

\section{Conclusion}

Nous espérons, par ces quelques réflexions et ces exemples, avoir jeté un peu de lumière sur les processus de compréhension humaine qui ne sont peut-être pas aussi insaisissables qu'il y paraît à première vue. Il semblerait que, pour comprendre des phrases d'une certaine opacité, on doive obligatoirement effectuer des opérations d'analyse et de décomposition d'éléments linguistiques complexes, et qu'il soit possible de les représenter de façon plus ou moins formelle. C'est ce que nous avons entrepris de faire, car les formalisations ont une grande valeur de démonstration, malgré leur caractère simplificateur.

Sur le plan pédagogique, nous avons voulu rendre plus explicites les notions traditionnelles de faux sens, contresens et non-sens. En remontant à la source de l'erreur et en offrant des outils de mesure de la gravité de l'erreur, les étudiants peuvent acquérir une meilleure conscience de leurs lacunes et trouver les remèdes appropriés.

Université de Montréal 


\section{Annexe}

As activists and supporters in the Democratic Party are already looking to the 1992 presidential elections, it is already a pretty safe bet that neither Michael Dukakis nor Jesse Jackson is going to be their candidate next time.

Dukakis has proven himself something of a political Martian. An activist from the left wing of the party put it this way: "Those people over in Massachussetts have never seen a smart Republican, and they were too arrogant to take advice from those of us who have. " Feeling in the Democrats' conservative wing is almost as bitter : "We can't win with Jesse ; we can't win without him ", said one of their number.

It is easy to be wise after an event. But the Jackson people can say with justification that if their man had been white, he might have pulled it off with what top adviser Bob Borosage of the Institute for Policy Studies calls his « updated version of the populist strain in the Democratic Party politics " - bringing out non-voters and the excluded with an assault on the "economic royalists. » Jackson's reception among white, blue-collar workers shows what can be done, says Borosage.

In the post-mortem that has already started, the Democrats' right wing will press for further moderation and accommodation of the social and foreign policy prejudices of Southern white males and "Reagan Democrats." A candidate for 1992 who fits that bill might be Sen. Sam Nunn or Sen. Albert Gore, who did well on Super Tuesday but proved to be no Crocodile Dundee in the jungles of New York.

from Michael White, "The Guardian ", London, in World Press Review, December 1988

\section{Références}

BEAUGRANDE, R. de (1980). Text, Discourse and Process (Towards a Multidisciplinary Science of Texts), vol. IV, dans la collection " Advances in Discourse Processes », New Jersey, Ablex Publ. 
BÉNARD, J.-P. et P. HORGUELIN (1979). Pratique de la traduction. Version générale, Montréal, Linguatech.

BOURBEAU, L. et J. LEHRBERGER (1988). Machine Translation : Linguistic Characteristics of MT Systems and General Methodology of Evaluation, vol. 15 de Linguisticae Investigationes Supplementa, Amsterdam, John Benjamins Publishing Company.

DECHERT, H. W. et U. SANDROCK (1986). "Thinking-Aloud Protocols ; the Decomposition of language processing " in V. COOK (ed), Experimental Approaches to Second Language Learning, Oxford Pergamon, 111-126.

DELISLE, J. (1984). L'Analyse du discours comme méthode de traduction; théorie et pratique, Éditions de l'Université d'Ottawa.

FILLMORE, C. (1968). "The Case for Case». Universals in Linguistic Theory, Bach \& Harms, Chicago, Reinhart and Winston, 1-90.

GOUADEC, D. (1974). Comprendre et traduire, Paris, Bordas.

JACKENDOFF, R. (1985). Semantics and Cognition, Cambridge, the MIT Press.

KINSTCH, W. et T.A. VAN DIJK (1975). «Comment on se rappelle et on résume des histoires". Langages, ${ }^{\circ}$ 40, déc. 1975, Didier, Larousse.

KINTSCH, W. et T.A. VAN DIJK (1983). Strategies of Discourse Comprehension, New York, Academic Press.

KRINGS, H.P. (1986). Was in den Köpfen von Übersetzern Vorgeht, Tübingen, Gunter Narr TBL.

LAROSE, R. (1989). Théories contemporaines de la traduction, Presses de l'université du Québec.

LÖRSCHER, W. (1987). Übersetzungsperformanz, Übersetzungsprozess und Übersetzungsstrategien, Habilitationsschrift, Universität Essen.

MAILLOT, J. (1969). La traduction scientifique et technique, Paris, Éditions Eyrolles.

MEL' ̌ UK, I. (1978). "Théorie de langage, théorie de traduction », Meta, Vol. XXIII, $\mathrm{n}^{\mathrm{o}} 4$, déc. 1978, pp. 271-302.

MEL'ČUK, I. (1981). «Meaning-Text Models : A Recent Trend in Soviet Linguistics ». Annual Review of Anthropology, vol. 10, pp. 27-62.

METZING, D. (éd.) (1980). Frame Conceptions and Text Understanding, Berlin et New York, Walter de Gruyter.

MINSKY, M. (1974). A Framework for Representing Knowledge, Memo 306, Cambridge, The MIT Press.

MOUNIN, G. (1976). Linguistique et traduction, Bruxelles, Dessart et Mardaga.

SABAH, G. (1988). L'intelligence artificielle et le langage, représentations des connaissances, Paris, Hermès.

SCHANK, R. et R. ABELSON (1977). Scripts, Plans, Goals and Understanding, Hillsdale, New Jersey, Lawrence Erlbaum. 
SEGUINOT, C. (Sous presse). "The Translation Process : An Experimental Study», in The Translation Process, Toronto, School of Translation, York University.

SELESKOVITCH, D. et M. LEDERER (1984). Interpréter pour traduire, Paris, Publications de la Sorbonne, Didier érudition.

SANDROCK, U. (1982). Thinking-Aloud Protocols (TAPs) - Ein Instrument zur Dekomposition des komplexen Prozesses "Übersetzen", Habilitationsschrift.

STEINER, G. (1975). After Babel, Aspects of Language and Translation, Londres, Oxford University Press.

TIERKKONEN-CONDIT, S. (1987). "Professional vs. Non-Professional Translation ", Paper read at the 8th Congress of Applied Linguistics, Sydney 16-21 August 1987.

VAN DIJK, T.A. (1977). Text and Context, Explorations in the Semantics and Pragmatics of Discourse, Londres et New York, Longman.

VINAY, J.P. et J. DARBELNET (1968). Stylistique comparée du français et de l'anglais, Montréal, Beauchemin. 